\title{
The Meningioma Vascularity Index: a volumetric analysis of flow voids to predict intraoperative blood loss in nonembolized meningiomas
}

\author{
Carlito Lagman, MD, ${ }^{1}$ Vera Ong, BS, ${ }^{1}$ Thien Nguyen, BS, ${ }^{1}$ Yasmine Alkhalid, BS, ${ }^{1}$ \\ John P. Sheppard, MS, ${ }^{1}$ Prasanth Romiyo, BS, ${ }^{1}$ Daniel Azzam, BS,, Giyarpuram N. Prashant, MD, ${ }^{1}$ \\ Reza Jahan, MD, ${ }^{3}$ and Isaac Yang, MD ${ }^{1,2,4-7}$

\begin{abstract}
Departments of ${ }^{1}$ Neurosurgery, ${ }^{2}$ Radiation Oncology, ${ }^{3}$ Radiological Sciences, ${ }^{4}$ Head and Neck Surgery, and the ${ }^{5} \mathrm{Jonsson}$ Comprehensive Cancer Center, David Geffen School of Medicine at the University of California, Los Angeles (UCLA), California; and ${ }^{6}$ Department of Neurosurgery and ${ }^{7}$ Los Angeles Biomedical Research Institute, Harbor-UCLA Medical Center, Torrance, California
\end{abstract}

\begin{abstract}
OBJECTIVE Meningiomas that appear hypervascular on neuroimaging could be amenable to preoperative embolization. However, methods for measuring hypervascularity have not been described, nor has the benefit of preoperative embolization been adjudicated. The objective of this study was to show a relationship between flow void volume (measured on MRI) and intraoperative estimated blood loss (EBL) in nonembolized meningiomas.
\end{abstract}

METHODS The authors performed volumetric analyses of 51 intracranial meningiomas (21 preoperatively embolized) resected at their institution. Through the use of image segmentation software and a voxel-based segmentation method, flow void volumes were measured on T2-weighted MR images. This metric was named the Meningioma Vascularity In$\operatorname{dex}(\mathrm{MVI})$. The primary outcomes were intraoperative EBL and perioperative blood transfusion.

RESULTS In the nonembolized group, the MVI correlated with intraoperative EBL when controlling for tumor volume ( $r$ $=0.55, p=0.002$ ). The MVI also correlated with perioperative blood transfusion (point-biserial correlation $\left[r_{p b}\right]=0.57, p=$ 0.001). A greater MVI was associated with an increased risk of blood transfusion (odds ratio [OR] 5.79, 95\% confidence interval [Cl] 1.15-29.15) and subtotal resection (OR 7.64, 95\% Cl 1.74-33.58). In the embolized group, those relationships were not found. There were no significant differences in MVI, intraoperative EBL, or blood transfusion across groups.

CONCLUSIONS This study clearly shows a relationship between MVI and intraoperative EBL in nonembolized meningiomas when controlling for tumor volume. The MVI is a potential biomarker for tumors that would benefit from embolization.

https://thejns.org/doi/abs/10.3171/2018.1.JNS172724

KEYWORDS embolization; meningioma; neuroimaging; oncology; Meningioma Vascular Index

I $\mathrm{N}$ select intracranial meningiomas, preoperative embolization may be appropriate. Currently, there is controversy about the indications for preoperative embolization, the interval between embolization and surgery, and the benefit of embolization in terms of surgical outcomes. ${ }^{3,7,8,12}$ It is also unclear what features of a meningioma are ideal for preoperative embolization. Some authors have suggested that hypervascular meningiomas could be amenable to preoperative embolization. ${ }^{6,13}$ To the best of our knowledge, efforts at radiologically quantifying hy- pervascularity of meningiomas have not been described, and hypervascularity reflected by the volume of flow voids has not been examined as a predictor of intraoperative estimated blood loss (EBL).

On neuroimaging, hypervascular meningiomas often have many flow voids and display the classic "sunburst" appearance, which reflects a large arterial pedicle and the vascular branches that radiate out from the feeding artery. Flow voids are the result of blood that courses through these vessels at velocities enough to produce a signal loss.

ABBREVIATIONS EBL = estimated blood loss; MMA = middle meningeal artery; $\mathrm{MVI}=$ Meningioma Vascular Index; $n-\mathrm{BCA}=n$-butyl cyanoacrylate; $\mathrm{PVA}=$ polyvinyl alcohol; $r_{p b}=$ point-biserial correlation; $v W F=$ von Willebrand factor.

SUBMITTED October 27, 2017. ACCEPTED January 10, 2018.

INCLUDE WHEN CITING Published online June 22, 2018; DOI: 10.3171/2018.1.JNS172724. 
The phenomenon is due to time-of-flight and spin-phase effects and is best visualized on T2-weighted sequences. ${ }^{14}$ In this paper we present a method of assessing the degree of hypervascularity in intracranial meningiomas using a volumetric analysis of flow voids on preoperative T2weighted MRI sequences. We named this novel metric the Meningioma Vascular Index (MVI). The aim of this study is to evaluate the MVI as a potential biomarker for meningiomas that may benefit from preoperative embolization, by showing that MVI is correlated with intraoperative EBL in nonembolized meningiomas.

\section{Methods \\ Study Protocol}

The medical records of consecutive patients who underwent craniotomy for tumor resection at the Ronald Reagan University of California, Los Angeles (UCLA) Medical Center between March 2013 and January 2017 were retrospectively reviewed to find patients who had preoperative imaging (CT and MRI [T1- and T2-weighted sequences]) with a histopathological diagnosis of meningioma (regardless of WHO grade). We excluded cases if there was only outside hospital imaging that could not be uploaded, or no T2-weighted imaging. In addition, recurrent meningiomas, cystic meningiomas, and those that were previously irradiated were not included. Fifty-one intracranial meningiomas met these criteria, including 21 that were preoperatively embolized. We used a 1.5-T scanner (Siemens) and a standard head coil for all MRI studies. Individual patient consent was not required for this study because we performed data collection retrospectively and excluded all patient identifiers.

\section{Segmentation Protocol}

The IRB approved the segmentation protocol. The files were completely de-identified and imported into an opensource image segmentation software, ITK-SNAP (version 3.6.0, ITK-SNAP). ${ }^{15}$ Prior to segmentation, the meningiomas were identified on T1-weighted images with Gd contrast and confirmed on T2-weighted images. We also reviewed T1-weighted MR images and CT scans to account for calcification of meningiomas that can also appear similar to flow voids. Calcified portions of the tumor were not segmented. We segmented flow voids on T2-weighted images and the procedure included three main steps: 1) identification of the vascular pedicle, often near the dural attachment; 2) segmentation of flow voids in consecutive axial slices with a brush size of 1 voxel; and 3) refinement of the original delineation (Fig. 1). The volume of the flow voids is automatically calculated in cubic millimeters $\left(\mathrm{mm}^{3}\right)$ by the software based on the number of voxels selected, and manually converted to cubic centimeters $\left(\mathrm{cm}^{3}\right)$ to generate the MVI.

In cases in which the vascular pedicle was not visible, we identified the base of the meningioma and reviewed consecutive slices until we recognized a flow void. We then segmented the flow void in a radial direction; the cutoff value for a flow void was 4 voxels. The second and third authors independently performed all segmentations (102 total segmentations); the first author reviewed and adjusted the segmentations as needed. Interobserver reliability was then tested across the observers. We measured the volumes of flow voids on axial T2-weighted sequences with 100 slices of 1-mm thickness. Additionally, we measured tumor volumes using a fully automated voxel-based segmentation method ${ }^{5}$ and investigated preoperative tumor volume and the skull base as potential confounders.

\section{Outcome Measures}

Authors who were uninvolved with the segmentation process collected the data. The primary outcomes were intraoperative EBL (as recorded on the operative note) and perioperative blood transfusion (documented in the blood bank report). Selection of patients for preoperative embolization depended on the senior author's (I.Y.) preference and practice. In general, the goal of preoperative embolization in each case was tumor blood flow reduction by eliminating 1 or more arterial feeders to the tumor. However, a multidisciplinary team evaluated each case for the appropriateness of and aggressiveness with which to perform embolization. Consideration of both visualized and potential external-to-internal carotid artery anastomoses and critical arterial supply to cranial nerves was undertaken before embolization.

When the interventional radiologist (R.J.) visualized no or minimal tumor blush or decided that no other feeding vessels could be safely embolized, the embolization procedure was concluded. We chose not to specify a cutoff threshold for reduction in tumor blush because only 7 procedural notes reported this value. We defined a successful embolization as minimal residual tumor blush with evidence of particle or coil embolization of the main arterial feeder and without evidence of vascular injury. For the embolization group, we noted the embolized vessel, embolization material, fluoroscopy time, and immediate complications. We deciphered the reduction in tumor blush and days from the embolization to the surgery from the interventional radiologist's notes. We deciphered the Simpson grade from the operative notes. Gross-total resection was defined as Simpson grade 1 or 2. Subtotal resection was defined as Simpson grade 3 or 4.

\section{Statistical Methods}

We assessed interobserver reliability using Cronbach's alpha. We performed standard tests for the comparisons of MVI across embolization groups and blood transfusion groups. To test for directional relationships between continuous-continuous and continuous-dichotomous variable sets we ran Pearson (r) and point-biserial correlations $\left(\mathrm{r}_{\mathrm{pb}}\right)$, respectively. We used the Mantel-Haenszel method ${ }^{11}$ to calculate the odds of experiencing the outcomes of interest based on a predetermined cutoff threshold for MVI. These tests were 2-tailed with a significance level of 0.05 set a priori. We used the SAS statistical program for all of the analyses.

\section{Results}

We included 51 intracranial meningiomas (21 preoperatively embolized) in the present study and summarize the patients in Tables 1 and 2. The most common vessels 

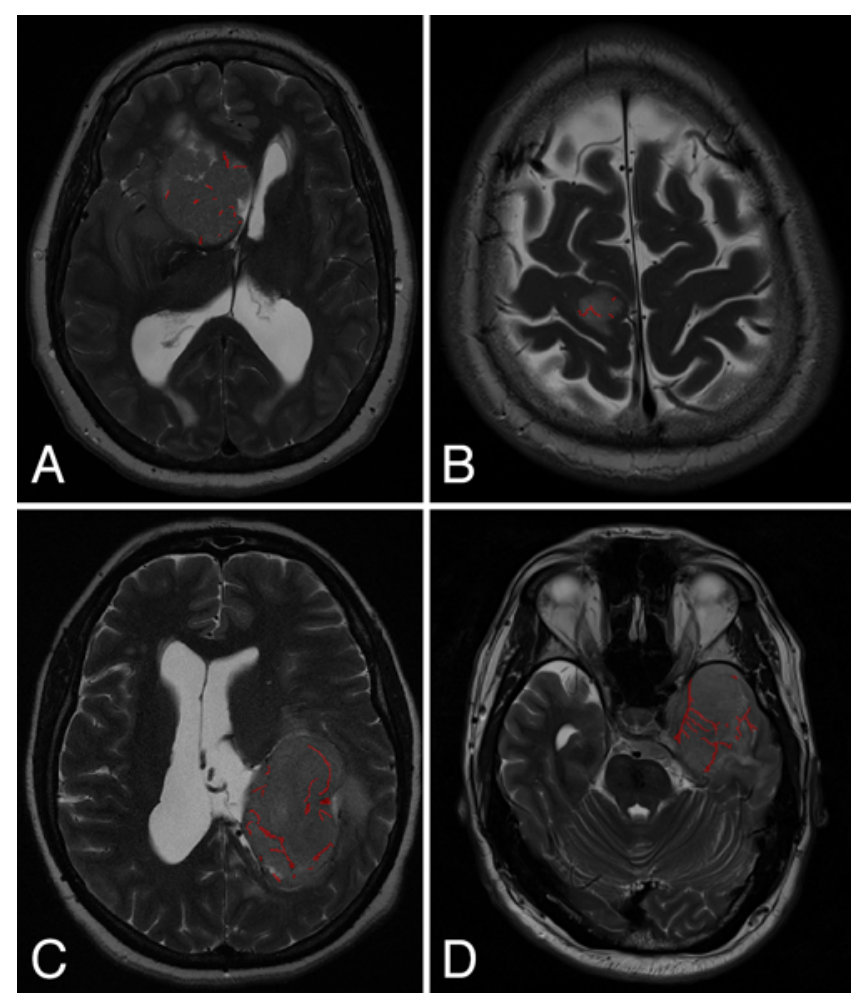

FIG. 1. MVI measured on axial T2-weighted MR images using the ITKSNAP software. A: MVI less than $2.5 \mathrm{~cm}^{3}$ and unsuccessful embolization. B: MVI less than $2.5 \mathrm{~cm}^{3}$ and successful embolization. C: MVI greater than $2.5 \mathrm{~cm}^{3}$ and unsuccessful embolization. D: MVI greater than $2.5 \mathrm{~cm}^{3}$ and successful embolization. Figure is available in color online only.

embolized were branches of the middle meningeal artery (MMA; 95\%). The most common embolisate used was polyvinyl alcohol (PVA; 76\%) often combined with $n$-butyl cyanoacrylate (n-BCA) and/or coils (62\%). The average fluoroscopy time $( \pm \mathrm{SD}$ ) was $34.30 \pm 18.79$ minutes (range 9.50-74.60 minutes). The percentage reduction in tumor blush was reported in only 7 patients; the average reduction in tumor blush was $52 \%$. There were no immediate complications in the 21 embolization procedures.

Cronbach's alpha for MVI was 0.89, which suggests good internal consistency across observers. Thus, we only used the first set of MVI values. The average MVI was $1.83 \mathrm{~cm}^{3}$ (mean 1.95, range $0.04-8.99 \mathrm{~cm}^{3}$ ) and did not differ when we compared embolized versus nonembolized groups ( 2.63 vs $1.27 \mathrm{~cm}^{3}, \mathrm{p}=0.16$ ). Figure 2 shows the relationship between MVI and intraoperative EBL for all patients and for the nonembolized group. In the nonembolized group, the MVI correlated with intraoperative EBL when controlling for tumor volume $(r=0.55, p=0.002)$. The MVI also correlated with perioperative blood transfusion $\left(\mathrm{r}_{\mathrm{pb}}=0.57, \mathrm{p}=0.001\right)$. A greater MVI was associated with an increased risk of blood transfusion (odds ratio [OR] 5.79, 95\% confidence interval [CI] 1.15-29.15) and subtotal resection (OR 7.64, 95\% CI 1.74-33.58). In the embolized group, those relationships were not found.

The average operative time was longer for embolized versus nonembolized groups (8.40 vs 13.84 hours, $\mathrm{p}=0.009)$. The intraoperative complication rate was $6 \%$,
TABLE 1. Summary of patients

\begin{tabular}{|c|c|}
\hline Variable & Value \\
\hline \multicolumn{2}{|l|}{ Age, yrs } \\
\hline Mean \pm SD & $58 \pm 13$ \\
\hline Range & $31-81$ \\
\hline \multicolumn{2}{|l|}{ Sex, n (\%) } \\
\hline Male & $14(27)$ \\
\hline Female & $37(73)$ \\
\hline \multicolumn{2}{|l|}{ Volumes $\left(\mathrm{cm}^{3}\right)$} \\
\hline Mean MVI \pm SD & $1.83 \pm 1.95$ \\
\hline Tumor \pm SD & $73 \pm 102$ \\
\hline Preop embolization, $\mathrm{n}(\%)$ & $21(41)$ \\
\hline Surgery, $\mathrm{n}(\%)$ & $51(100)$ \\
\hline Mean op time $\pm S D$, hrs & $11 \pm 7$ \\
\hline Mean EBL $\pm \mathrm{SD}, \mathrm{ml}$ & $239 \pm 176$ \\
\hline \multicolumn{2}{|l|}{ Simpson grade, $n(\%)$} \\
\hline 1 & $27(53)$ \\
\hline 2 & $4(8)$ \\
\hline 3 & $4(8)$ \\
\hline 4 & $15(29)$ \\
\hline 5 & $1(2)$ \\
\hline \multicolumn{2}{|l|}{ WHO grade, $n(\%)$} \\
\hline 1 & $36(71)$ \\
\hline II & $15(29)$ \\
\hline RBCT, n (\%) & $6(12)$ \\
\hline
\end{tabular}

$\mathrm{RBCT}=$ red blood cell transfusion .

which included intraoperative cerebral edema $(n=1)$, transient intraoperative hypotension $(\mathrm{n}=1)$, and temporary loss of somatosensory evoked potentials $(n=1)$. The average MVI was greater for the subtotal resection group versus the gross-total resection group (2.41 vs $1.46 \mathrm{~cm}^{3}, \mathrm{p}$ $=0.04)$. In a multivariate logistic regression model neither MVI nor tumor volume predicted subtotal resection (OR $1.26,95 \%$ CI $0.89-1.76$, and OR $1.00,95 \%$ CI $1.00-1.01$ ). An MVI greater than or equal to $2.5 \mathrm{~cm}^{3}$ was an independent predictor of subtotal resection (OR 7.64, 95\% CI 1.74-33.58); this remained significant when controlling for tumor volume (OR 10.62, 95\% CI 1.87-60.10) and the skull base (OR 7.75, 95\% CI 1.75-34.32).

There were 6 patients (12\%) transfused with red blood cells: 2 intraoperatively and 4 postoperatively. When we compared embolized versus nonembolized meningiomas, the transfusion rates were similar $(14 \%$ vs $10 \%, \mathrm{p}=0.68)$. The average number of units transfused was 1 unit (range 1-3 units). The average MVI was greater for the transfusion group versus the nontransfusion group (3.76 vs 1.57 $\left.\mathrm{cm}^{3}, \mathrm{p}=0.005\right)$. MVI was correlated with blood transfusion $\left(r_{\mathrm{pb}}=0.36, \mathrm{p}=0.009\right)$. In a multivariate logistic regression model, MVI predicted blood transfusion (OR 1.63, 95\% CI 1.04-2.57) but preoperative tumor volume did not (OR $1.02,95 \%$ CI 1.00-1.03). An MVI greater than or equal to $2.5 \mathrm{~cm}^{3}$ was an independent predictor of blood transfusion (OR 4.00, 95\% CI 1.01-15.78); this was no longer significant when controlling for tumor volume (OR 5.79, $95 \%$ CI 
TABLE 2. Summary of patients who underwent preoperative embolization

\begin{tabular}{|c|c|c|c|c|c|c|c|c|c|}
\hline Age (yrs), Sex & Meningioma & $\mathrm{MVI}\left(\mathrm{cm}^{3}\right)$ & Artery & Material & Op Time (hrs) & $\mathrm{EBL}(\mathrm{ml})$ & Simpson Grade & WHO Grade & RBCT \\
\hline $43, \mathrm{~F}$ & Anterior clinoid & 1.97 & MMA & Coil & 14 & 400 & 4 & $\|$ & No \\
\hline $48, F$ & Petroclival & 3.76 & MMA & Coil & 11 & 200 & 4 & I & No \\
\hline $44, F$ & Parietal, parasagittal & 0.38 & MMA & $n-B C A$ & 8 & 250 & 1 & II & No \\
\hline $53, \mathrm{M}$ & Frontal-parietal & 0.19 & MMA & $n-B C A$ & 13 & 200 & 1 & I & No \\
\hline $33, \mathrm{M}$ & Middle fossa & 0.79 & MMA & $n-B C A$ & 22 & 200 & 2 & I & No \\
\hline $73, F$ & Sphenoid wing & 0.74 & MMA, IMAX & PVA & 4 & 400 & 4 & I & No \\
\hline $60, M$ & Bifrontal & 7.68 & MMA, STA & PVA & 10 & 300 & 1 & II & No \\
\hline $79, F$ & Parietal-occipital & 3.30 & MMA, STA & PVA & 10 & NR & 4 & I & No \\
\hline $60, F$ & Temporal-occipital & 8.99 & MMA & PVA, coil & 3 & 100 & 1 & 1 & No \\
\hline $66, F$ & Frontal & 0.42 & MMA, IMAX & PVA, coil & 2 & 100 & 1 & I & No \\
\hline $46, M$ & Parafalcine & 3.28 & MMA & PVA, coil & 21 & 350 & 3 & 1 & No \\
\hline $58, F$ & Parafalcine & 0.57 & MMA & PVA, coil & 8 & 200 & 1 & I & No \\
\hline $65, \mathrm{~F}$ & Frontal & 1.06 & IMAX, MMA & PVA, coils & 10 & 200 & 1 & I & No \\
\hline $74, \mathrm{~F}$ & Sphenoid wing & 5.13 & MMA & PVA, coils & $<1$ & 900 & 4 & 1 & Yes \\
\hline $46, M$ & Anterior skull base & 5.05 & $\begin{array}{l}\text { MMA, AMA, } \\
\text { IMAX }\end{array}$ & PVA, coils & 5.6 & 250 & 4 & 1 & No \\
\hline $81, \mathrm{~F}$ & Frontal & 1.45 & MMA & PVA, coils & $<1$ & 150 & 3 & II & No \\
\hline $79, \mathrm{~F}$ & Cerebellar & 0.29 & Occipital & PVA, n-BCA & 4 & 200 & 3 & I & No \\
\hline $33, \mathrm{M}$ & Frontal & 1.68 & MMA & PVA, n-BCA & 6 & 300 & 1 & 1 & No \\
\hline $45, \mathrm{~F}$ & Temporal & 0.04 & $\begin{array}{l}\text { MMA, MDTA, } \\
\text { ADTA }\end{array}$ & PVA, n-BCA & 8 & 300 & 2 & I & No \\
\hline $53, F$ & Frontal-orbital & 2.29 & MMA & PVA, n-BCA & 12 & 500 & 1 & 1 & Yes \\
\hline $74, \mathrm{~F}$ & Sphenoid wing & 6.15 & MDTA, MMA & PVA, n-BCA, coil & 4.8 & 250 & 4 & I & Yes \\
\hline
\end{tabular}

ADTA = anterior deep temporal artery; AMA = accessory meningeal artery; IMAX = internal maxillary artery; MDTA = middle deep temporal artery; NR = not reported; STA = superficial temporal artery.

0.75-44.72) but remained significant when controlling for the skull base (OR 8.78, 95\% CI 1.31-59.95).

\section{Discussion}

In the published literature, there have been many attempts at correlating preoperative imaging and intraoperative EBL. ${ }^{1,2,4,9,10,12}$ Lü investigated several imaging features in meningiomas and found that origin, volume, and blood vessel or venous sinus involvement correlated with intraoperative EBL..$^{10}$ In addition, the authors found that origin, volume, and blood vessel or venous sinus involvement independently predicted intraoperative red blood cell transfusion. In the present study, we focused on hypervascularity of meningiomas, as quantified by the MVI, and showed that MVI was associated with intraoperative EBL and blood transfusion in nonembolized meningiomas.

Prior studies investigated the quantitative analysis of meningioma vascularity using molecular markers such as von Willebrand factor (vWF) and hypoxia-inducible factor-1. Karsy et al. identified vWF as a significant predictor of EBL in a univariate model. ${ }^{9}$ Unfortunately, vWF is not routinely ordered in patients with meningiomas, whereas MRI is almost always required for these patients. Nguyen et al. examined $\mathrm{T} 1$ postcontrast intensity as a predictor of blood loss in meningiomas (with no patients having undergone preoperative embolization) and reported that the $\mathrm{T} 1$ index (the average $\mathrm{T} 1 \mathrm{C}+$ intensity across the tumor nor- malized to a value at the basilar artery) and tumor volume were significant predictors of EBL. ${ }^{12}$

A previously published study compared outcomes related to preoperative embolization of meningiomas and reported that EBL was higher in the embolized group as compared to the nonembolized group (410 vs $315 \mathrm{ml}$, p $=0.007) \cdot{ }^{13}$ We observed a similar trend, but our sample size for embolized meningiomas was smaller (21 vs 177 embolized meningiomas). That data point suggests that embolization is associated with greater blood loss and that we should not perform the procedure; however, the reality is that this is likely due to patient selection and that neurosurgeons are more likely to recommend embolization for tumors assumed to be more vascular, difficult, or challenging for early devascularization. Based on the results of that study, the authors recommended consideration of preoperative angiography and embolization for: 1) meningiomas in areas of eloquence; 2) meningiomas with extensive intratumoral calcifications; 3) meningiomas greater than $3-4 \mathrm{~cm}$ in diameter with at least $50 \%$ of the vascular supply being accessible branches of the external carotid artery; and 4) hypervascular meningiomas or those with surgically inaccessible vascular supply. ${ }^{13}$

The debate about the interval between embolization and surgery continues. ${ }^{3,78}$ Chun et al. evaluated outcomes following preoperative embolization of meningiomas and found that intraoperative EBL was greater for patients who underwent resection within a day after embolization when 

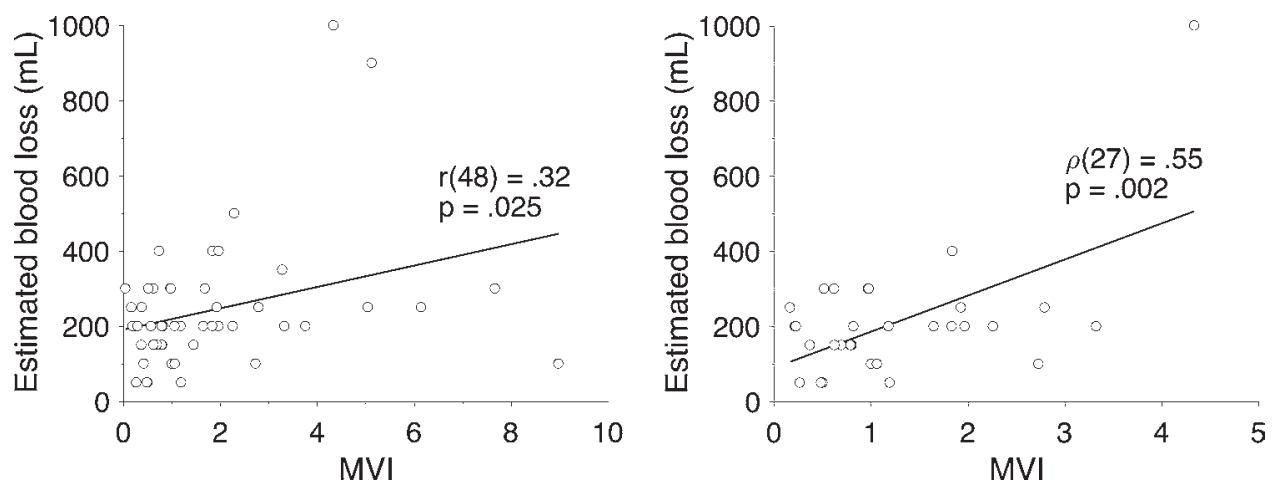

FIG. 2. Scatter plots of MVI versus intraoperative EBL for all cases (left) and nonembolized cases only (right). $r=$ Pearson's correlation coefficient. $\rho=$ Spearman's partial correlation controlling for preoperative tumor volume.

compared to patients who underwent resection more than a day after embolization. ${ }^{3}$ Thus, the authors of that study suggested that we should delay resection for more than 24 hours. Our practice is to perform resection of meningiomas 24 hours after embolization. There are no published guidelines to dictate this practice; the reason for this is logistical. At our institution, preoperative embolization is typically performed in the morning and completed at approximately noon. We prefer to begin surgery also in the morning (and not the afternoon), and therefore perform surgery the following day.

\section{Key Findings and Implications}

In this study, we presented a novel method to measure hypervascularity of intracranial meningiomas using a voxel-based analysis of flow voids. We named this novel measurement the Meningioma Vascularity Index, or MVI. We clearly show a relationship between MVI and intraoperative EBL in nonembolized meningiomas when controlling for tumor volume. Thus, our findings support the recommendation for embolization of hypervascular meningiomas by Raper et al. ${ }^{13}$ Perhaps more important is that this work shows that measuring hypervascularity can be performed using noninvasive neuroimaging with standard sequences. The image segmentation software we used is available open-source, which would make a multicenter study possible. Such a study could test the index developed here in a larger cohort to enable more definitive conclusions and a broader use of the procedure.

\section{Limitations}

Our study has several limitations. We could only analyze T2-weighted images that could be retrieved and imported into the image segmentation software. Segmentation of flow voids on T2 does not account for calcification that can also look like flow voids. Calcification is a major confounder using T2-weighted images. CT imaging was not available for every patient to rule out calcification. Nevertheless, we suspected partially calcified meningiomas in 17 patients. CT imaging was available in all of those patients, and 4 had partially calcified meningiomas. Calcified meningiomas were not completely excluded from the study, however, calcified portions of the tumor were not segmented.

Initially, we attempted an automated approach to seg- ment the flow voids, but realized that the coverage of the segmentation was minimal even for meningiomas fed by relatively high-caliber vessels. We used a manual approach instead. The advantages of this approach are greater coverage and standardization of the protocol. The disadvantages include more time and increased variability. Advances in image segmentation software will likely make automatic segmentation of flow voids possible in the near future. To the best of our knowledge, there are no objective measures to identify highly branched vessels within meningiomas, so a subgroup analysis for meningiomas with many branching vessels would inevitably introduce additional bias. Thus, we did not validate the use of MVI specifically for highly branched vessels, but the majority of meningiomas included in the present study were considered highly branched.

We also segmented all visible flow voids, which included deep feeding arteries that are not necessarily amenable to embolization. There are several factors that determine whether embolization of a vessel is possible, which were not controlled for. These factors include, but are not limited to, 1) the region supplied by the arterial feeder, 2) the presence of collaterals, and 3) technical difficulties in accessing low-caliber vessels and/or vessels with an acute angle of origin. Notwithstanding those issues, the calculated MVI had moderate correlations with intraoperative findings and predicted extent of resection that ignores large vessel encasement. Also, convexity meningiomas supplied exclusively by a single MMA feeder in the same study as a petrous face meningioma - that may recruit clival, ascending pharyngeal, posterior meningeal supply, and further parasitize pial feeders-could be irrelevant to the study.

We also could not standardize the embolization procedures, so there were variabilities in technique that could not be accounted for. Additionally, the choice of embolization material was operator dependent, and therefore, inconsistent across patients. In this study, a response rate of greater than $33 \%$ (7 of 21 cases with reported data) limits interpretation of our results and is a reporting bias. Although the senior authors (R.J. and I.Y.) provided the endovascular and open surgical care for all patients, respectively, interoperator variability is a general critique of evidence in preoperative embolization.

It is also important to emphasize that we investigated a single radiological feature that represents the most dis- 
tal endpoint of an embolization procedure, i.e., the blood supply to the tumor. Moreover, flow voids do not really represent true tumor vascularity, which could be better assessed by dynamic susceptibility contrast perfusion or other advanced MRI methods. Unfortunately, those studies are not routinely ordered for patients diagnosed with meningiomas, and therefore could not be segmented retrospectively. We chose to use the most common MRI sequences ordered to increase the broad generalizability and utility of our method. This is especially helpful in smaller hospitals, which may not have the resources to perform advanced studies.

Perhaps the most critical limitations are the small sample size and that the reported EBL remains a subjective outcome measure with high interobserver variability. Furthermore, blood transfusion remains a subjective measure because there is integration of objective (such as trends in hemoglobin and hematocrit, and/or vital sign indicators, which are also subject to clinical interpretation) and subjective (impression of EBL) data in clinical practice.

\section{Conclusions}

Although the appropriateness of preoperative embolization of an intracranial meningioma can only be determined through cerebral angiography and clinical judgment, we clearly show a relationship between MVI and intraoperative EBL in nonembolized meningiomas after controlling for tumor volume. The study is the first to describe a radiological method for quantifying hypervascularity and to assess its predictive value. We encourage continued investigation in a larger prospective series with comparison to more advanced imaging methods.

\section{Acknowledgments}

Carlito Lagman is supported by the Tina and Fred Segal Benign Brain Tumor and Skull Base Surgery Research Fellowship. Thien Nguyen and John Sheppard are supported by the David Geffen Medical Scholarship. Yasmine Alkhalid is supported by The Medical College of Georgia Medical Scholars Program. Isaac Yang is supported by the UCLA Visionary Ball Fund Grant, Eli and Edy the Broad Center of Regenerative Medicine and Stem Cell Research UCLA Scholars in Translational Medicine Program Award, Jason Dessel Memorial Seed Grant, UCLA Honberger Endowment Brain Tumor Research Seed Grant, and Stop Cancer (US) Research Career Development Award.

\section{References}

1. Ali R, Khan M, Chang V, Narang J, Jain R, Marin H, et al: MRI pre- and post-embolization enhancement patterns predict surgical outcomes in intracranial meningiomas. J Neuroimaging 26:130-135, 2016

2. Bander ED, Singh H, Ogilvie CB, Cusic RC, Pisapia DJ, Tsiouris AJ, et al: Endoscopic endonasal versus transcranial approach to tuberculum sellae and planum sphenoidale meningiomas in a similar cohort of patients. J Neurosurg 128:40-48, 2018

3. Chun JY, McDermott MW, Lamborn KR, Wilson CB, Higashida R, Berger MS: Delayed surgical resection reduces intraoperative blood loss for embolized meningiomas. Neurosurgery 50:1231-1237, 2002

4. Coroller TP, Bi WL, Huynh E, Abedalthagafi M, Aizer AA,
Greenwald NF, et al: Radiographic prediction of meningioma grade by semantic and radiomic features. PLoS One 12:e0187908, 2017

5. Gaonkar B, Macyszyn L, Bilello M, Sadaghiani MS, Akbari $\mathrm{H}$, Atthiah MA, et al: Automated tumor volumetry using computer-aided image segmentation. Acad Radiol 22:653661,2015

6. Hu YC, Newman CB, McDougall CG, Albuquerque FC: Embolization of tumors: brain, head, neck, and spine, in Quiñones-Hinojosa A (ed): Schmidek and Sweet: Operative Neurosurgical Techniques, ed 6. Philadelphia: Elsevier, Vol 1, pp 1065-1078

7. James RF, Kramer DR, Page PS, Gaughen JR Jr, Martin LB, Mack WJ: Strategic and technical considerations for the endovascular embolization of intracranial meningiomas. Neurosurg Clin N Am 27:155-166, 2016

8. Kai Y, Hamada J, Morioka M, Yano S, Todaka T, Ushio $\mathrm{Y}$ : Appropriate interval between embolization and surgery in patients with meningioma. AJNR Am J Neuroradiol 23:139-142, 2002

9. Karsy M, Burnett B, Di Ieva A, Cusimano MD, Jensen RL: Microvascularization of Grade I meningiomas: effect on tumor volume, blood loss, and patient outcome. J Neurosurg 128:657-666, 2018

10. Lü J: Correlation between preoperative imaging features and intraoperative blood loss of meningioma: a new scoring system for predicting intraoperative blood loss. J Neurosurg Sci 57:153-161, 2013

11. Mantel N, Haenszel W: Statistical aspects of the analysis of data from retrospective studies of disease. J Natl Cancer Inst 22:719-748, 1959

12. Nguyen HS, Janich K, Doan N, Patel M, Li L, Mueller W: Extent of T1+C intensity is a predictor of blood loss in resection of meningioma. World Neurosurg 101:69-75, 2017

13. Raper DM, Starke RM, Henderson F Jr, Ding D, Simon S, Evans AJ, et al: Preoperative embolization of intracranial meningiomas: efficacy, technical considerations, and complications. AJNR Am J Neuroradiol 35:1798-1804, 2014

14. Vlaardingerbroek MT, Boer JA: Magnetic Resonance Imaging: Theory and Practice, ed 3. Berlin: Springer, 2003

15. Yushkevich PA, Piven J, Hazlett HC, Smith RG, Ho S, Gee JC, et al: User-guided 3D active contour segmentation of anatomical structures: significantly improved efficiency and reliability. Neuroimage 31:1116-1128, 2006

\section{Disclosures}

The authors report no conflict of interest concerning the materials or methods used in this study or the findings specified in this paper.

\section{Author Contributions}

Conception and design: Lagman, Jahan. Acquisition of data: Lagman, Ong, Nguyen, Alkhalid, Sheppard, Romiyo, Azzam. Analysis and interpretation of data: Yang, Lagman, Ong, Nguyen, Alkhalid, Sheppard, Romiyo, Azzam, Prashant. Drafting the article: Lagman, Ong, Nguyen, Alkhalid, Sheppard, Romiyo, Azzam, Prashant, Jahan. Critically revising the article: all authors. Reviewed submitted version of manuscript: all authors. Approved the final version of the manuscript on behalf of all authors: Yang. Statistical analysis: Lagman, Romiyo. Administrative/technical/ material support: Yang, Prashant, Jahan. Study supervision: Yang, Lagman, Prashant, Jahan.

\section{Correspondence}

Isaac Yang: University of California, Los Angeles, CA. iyang@ mednet.ucla.edu. 